\title{
Pattern of Hepatitis A Virus Epidemiology in Nursing Students and Adherence to Preventive Measures at Two Training Wards of a University Hospital
}

\author{
Marcello Campagna, ${ }^{1,}$ Noemi Maria Mereu, ${ }^{1}$ Lucia Mulas, ${ }^{2}$ Roberta Pilia, ${ }^{1}$ Maria Francesca Piazza, ${ }^{1}$ \\ Laura Spada, ${ }^{1}$ Alberto Lai, ${ }^{1}$ Igor Portoghese, ${ }^{1}$ Maura Galletta, ${ }^{1}$ Giuseppina Masia, ${ }^{1}$ Angelo Restivo, ${ }^{3}$ \\ Paolo Mura, ${ }^{4}$ Gabriele Finco, ${ }^{4}$ and Rosa Cristina Coppola ${ }^{1}$ \\ ${ }^{1}$ Department of Public Health, Clinical and Molecular Medicine, University of Cagliari, Cagliari, Italy \\ ${ }^{2}$ Azienda Ospedaliero-Universitaria, Policlinico Universitario, Monserrato, Cagliari, Italy \\ ${ }^{3}$ Department of Surgery, Colorectal Surgery Center, University of Cagliari, Cagliari, Italy \\ ${ }^{4}$ Anesthesia and Intensive Care Department, Pain Therapy Service, Department of Medical Sciences, University of Cagliari, Cagliari, Italy \\ "Corresponding author: Marcello Campagna, Marcello Campagna, Department of Public Health, Clinical and Molecular Medicine, University of Cagliari, Cagliari, Italy. Tel: \\ +39-0706754441, E-mail: mcampagna@unica.it
}

Received 2015 November 12; Accepted 2015 November 25.

\begin{abstract}
Background: Nursing students can be exposed to patients with hepatitis A virus (HAV) and can represent a vehicle of transmission both for health personnel, patients and relatives.

Objectives: The aim of this study was to assess the risk of HAV infection in nursing students during their internship.

Patients and Methods: A seroprevalence survey on HAV infection was performed on nursing students at the Cagliari universityhospital, together with the assessment of the compliance to preventive measures to decrease the risk of infection during their internship. Blood specimens were obtained from 253 students. All serum samples were tested for anti-HAV antibodies (IgG) by the enzymelinked immunosorbent assay (ELISA). Compliance to preventive measures was recorded by trained personnel.

Results: Overall HAV seropositivity in nursing students (mean age 24, range 17 - 45 years) was 3\%. Compliance to preventive measures was not uniform ( $6 \%-76 \%)$ and extremely low in some specific measures targeted to decrease the oral-fecal transmission.

Conclusions: The high proportion of susceptible nursing students can contribute to an increase in the risk of nosocomial transmission, especially when specific preventive measures are not completely applied. Nursing education packages, before starting medical internship, should be implemented in order to increase their compliance to preventive measures, especially in wards at higher risk. Vaccination should be considered in wards at higher risk.
\end{abstract}

Keywords: Nursing Students, Hepatitis A, Epidemiology, herd immunity, Preventive Measures

\section{Background}

Hepatitis A is an infectious disease caused by an RNA virus with oral-fecal transmission. The prevalence of infection is generally low during childhood and quickly increases during youth and between young adults. About 1.5 million clinical cases of Hepatitis A occur per year, yet the infection rate is probably higher (1). The prevalence is strongly tied to socioeconomic development and standards of hygiene. European mediterranean countries have classically been considered as areas of moderate endemicity for hepatitis A virus (HAV), where infection occurs mainly in children and young adults (2). However, epidemiological surveys carried out in the last few years in southern European countries showed a marked decrease in the prevalence of antibodies against HAV and a change in the epidemiological pattern of HAV infection (2). The Italian integrated epidemiological system for acute viral hepatitis surveillance (SEIEVA) reports that the incidence of HAV has declined from 10/100000 in 1985 to 3.6/100000 in 2004, and to $1.1 / 100000$ in 2010 , with an increase during 1996 - 1997 corresponding to a large outbreak, which occurred in two regions of southern Italy (Apulia and Campania). Epidemiological patterns vary among different regions within Italy, with low endemicity areas in central and northern regions and intermediate endemicity in southern and insular regions (3). A recent study conducted in Sardinia confirmed a marked downward trend of prevalence for antibodies against hepatitis A virus during the last 20 years $(4)$.

Due to the decrease in herd immunity, the identification of risk factors and groups at increased risk of infection is essential to implement efficacious preventive measures targeted to decrease the risk of HAV.

Several epidemiological surveys have shown an in- 
creased risk of HAV infection in the health personnel in comparison with the general population because of nosocomial exposure $(5,6)$. Moreover, health personnel can represent a vehicle of transmission to patients with clinical conditions and are thus at a higher risk of severe complications. Nevertheless, there is strong evidence that hand hygiene and adoption of standard and contact precautions can significantly decrease the risk of nosocomial oralfecal transmission of biological agents; non-compliance to these preventive practices remains a major problem in hospital settings (7-9).

Both in developed and developing countries, it is known that health professionals encounter numerous difficulties in adherence to hand hygiene practices (10). For example, a significant association has been found between workload, infections and poor adherence to hand hygiene practices. A recent study (11) highlighted that work overload was one of numerous barriers limiting compliance with hygiene procedures. Moreover, another relevant study showed that during understaffed and overcrowded period, the adherence to hand hygiene practices before device contact was $25 \%$, while at the end of this period it rose to $70 \%$ (12).

Among health personnel, nursing students can be exposed to patients with HAV infection during their bachelor degree (13), due both to the lack of immunity and because of their inexperience in performing preventive measures.

The aim of this study was to assess the serological status of HAV among nursing students, which potentially could be exposed to HAV infection, and compliance of healthcare staff to preventive measures in the wards where students were employed during their internship.

\section{Objectives}

The aim of this study was to assess the risk of HAV infection in nursing students during their internship.

\section{Patients and Methods}

\subsection{Study Population}

The study was conducted from September 2013 to January 2015. Nursing students attending the second year of their bachelor degree at the university of Cagliari, ranging in age from 19 to 45 years, were informed about the study aim and invited to participate. An inclusion criterion was to be involved in an internship in a hospital as a nursing student. All the involved students agreed to participate in the study.

\subsection{Serological Assays}

Based on an annual routine workplace health surveillance program (mandatory under the Italian health and safety law decree 81/08), an initial venous blood specimen ( $3 \mathrm{~mL}$ ) was obtained on a voluntary basis for specific antibody serum. The samples were tested for anti-HAV IgG, by a commercially available micro particle enzyme immunoassay (MEIA; AxSYM-HAVAB 2.0, ABBOTT). The cutoff values were established according to the manufacturer's instructions. As established by the Italian drug agency statement on the 20 March 2008 (guidelines on the classification and management of observational studies on drugs, official gazette of the Italian republic, ranked series general, $\mathrm{N}^{\circ} 76$ March 312008 ) the study protocol was notified to the competent institutional review board and the ethics committee of the Cagliari university hospital. Due to the observational nature of the study, in absence of any additional invasive procedure beyond the routine mandatory health screening protocol, and in the absence of any involvement of therapeutic medication, no formal approval was required.

\subsection{Compliance to Preventive Measures}

A panel of expert health advisors conducted a 15-month observation campaign at four intensive care units (ICUs) and in one colorectal surgical unit (SU) in which nursing students performed their internship. Compliance by physicians, nurses, and nurse aid to the world health organization (WHO) hand hygiene $(\mathrm{HH})$ criteria and to the correct use of gloves and standard precautions application, was randomly recorded.

\subsection{Statistical Analysis}

Age-specific and gender seroprevalence rates were computed. Chi-square $\left(\mathrm{X}^{2}\right)$ test was applied for the association between categorical variables. Statistical significance was set at $\mathrm{P}=0.05$. Both $95 \%$ confidence interval $(\mathrm{CI})$ and the poisson confidence interval were used when the number of observations was lower than ten. Statistical analysis was performed using the SPSS software (version 19; SPSS Inc., Chicago IL, USA).

\section{Results}

A total of 253 nursing students were enrolled in the study. The results showed that none of them was affected or suspected of hepatic disease. The participants included 184 (73\%) females and 69 (27\%) males. The mean age was 24 years (SD 7.6 minutes 19, max 45 years); 87\% (219/253) were aged 19 to 28 years, $10 \%$ (26/253) were aged 29 to 38 years and $3 \%$ (8/253) were aged $>38$ years. Seroprevalence of anti 
HAV antibodies was overall 3\% (Table 1). No significant difference was found regarding gender and age.

\begin{tabular}{|c|c|c|c|}
\hline Age Range, Y & $\begin{array}{l}\text { Total Number } \\
\text { of Nursing } \\
\text { Students } \\
\text { Positive/Tested, } \\
\%, 95 \% \text { CI }\end{array}$ & $\begin{array}{c}\text { Female, } \\
\text { Positive/Tested, } \\
\%\end{array}$ & $\begin{array}{c}\text { Male, } \\
\text { Positive/Tested, } \\
\%\end{array}$ \\
\hline $19-28$ & $\begin{array}{c}4 / 219(1.83), 0.05- \\
3.6\end{array}$ & $3 / 158(1.90)$ & $1 / 61(1.64)$ \\
\hline $29-38$ & $\begin{array}{c}4 / 26(15.38), 1.52- \\
29.25\end{array}$ & $4 / 18(22.22)$ & $0 / 8(0.00)$ \\
\hline$\geq 39$ & 0 & $0 / 8(0.00)$ & $0 / 0(0.00)$ \\
\hline Total & $\begin{array}{c}8 / 253(3.16), 1.01- \\
5.32\end{array}$ & $7 / 184(3.80)$ & $1 / 69(1.45)$ \\
\hline
\end{tabular}

Table 2 presents the observations in compliance with hand hygiene procedures and standard precautions during both the intensive care and colorectal surgical units stratified for job tasks. The results revealed that compliance was higher at ICUs if compared to the SU. Nurse aide had higher compliance rates than nurses and physicians at the ICU, yet not in SU. Hand hygiene procedures were correctly applied equal or less than $30 \%$ in all the units, except for nurse aide at the ICUs.

\section{Discussion}

Our findings showed that the HAV rate of seroprevalence among nursing students ranged between 1\% and 15\% in the age groups from 19 to 28 years, and from 29 to 38 years, respectively. The seroprevalence rate was relatively low compared with a recent study conducted in the same area in 2008, which showed seroprevalence ratios of $7.3 \%$ in the age group from 20 to 29 years, and $24.2 \%$ in the age group of 30 to 39 years (4).

The dramatic decrease in HAV seroprevalence in the previous recent years resulted in a lack of protective herd immunity. Moreover, decreasing rate of HAV infection in children and young adults increases the risk of infection in adults $(8,9)$ with possible serious and clinically severe complications, that are more common in older age groups (4).

Nursing students are at high risk for nosocomial transmission of HAV infection because of the lack of herd immunity and the possible exposure to HAV-infected patients during the clerkship. Moreover, our study showed an inadequate compliance by staff to preventive measures assessed in the units in which nurse students performed their internship. This critical factor can contribute to the increase in the risk of transmission of HAV to hypersusceptible patients (i.e. patient with chronic lung, liver and kidney disease, asplenia, heart disease and weakened immune system, HIV positive patients, and pregnant women) and colleagues.

This study showed a general lack of compliance to fundamental preventive measures for the management of healthcare-associated infections (HAIs). The differences among the involved wards could be due to several aspects. The higher compliance to hand hygiene at ICUs (e.g. before direct contact with the patient; after direct contact with the patient; after removing the gloves; and before using an invasive device for patient care) could be due to the fact that the clinical conditions of patients at intensive care units are generally more critical, and the operators perceive a higher risk associated with poor adherence to hand hygiene $(\mathrm{HH})$.

On the contrary, with regards to standard precautions, the SU adheres better than ICUs (e.g., use of the same gloves to assist more than one patient; and discharge gloves after touching environmental surfaces in proximity of the patient, including medical equipment) probably because in this case the operators are more often in contact with non-intact skin and this condition causes a major perception of risk. In this scenario, nursing students may be at particular risk also because of their inexperience. Nursing students should receive specific training on the risk of infections in hospital settings before starting internship together with a continuous improvement of health personnel at the units selected for the internship. Research has widely shown that the increase of $\mathrm{HH}$ adherence is related to the reduction of bacterial resistance and low HAI rates. This is the reason why the center for disease control (CDC) classifies hand hygiene as the most important measure in the control of hospital infections. In fact, a rigorous $\mathrm{HH}$ intervention can prevent about $40 \%$ of all nosocomial infections (14). Thus, compliance with standard precautions is a key point to decrease HAIs.

Moreover, this study highlighted an important aspect, which should be considered when training nurses. In effect, clinical placements should provide to the students a real-world environment in which theory translates into practice, thus developing skills and attitudes toward the nursing profession (15). In this sense, as clinical learning may become the first source of exposure to the risk of infectious diseases with potential consequences on students' psychological wellbeing, all healthcare professionals should serve as good examples for young students regarding compliance with the best clinical practice.

This study had some limitation. The observational method was considered by many authors as the gold standard for assessing hand hygiene compliance. However, the 
Table 2. Compliance to Hand Hygiene Procedures and Standard Precautions Stratified for Job Tasks in Internship Wards ${ }^{\mathrm{a}}$

\begin{tabular}{|c|c|c|c|c|c|c|}
\hline \multirow[t]{2}{*}{ Job Tasks } & \multicolumn{2}{|c|}{ ICU } & \multirow[t]{2}{*}{$\%(95 \% \mathrm{CI})$} & \multicolumn{2}{|c|}{ su } & \multirow[t]{2}{*}{$\%(95 \% \mathrm{CI})$} \\
\hline & Observations & Adhesions & & Observations & Adhesions & \\
\hline \multicolumn{7}{|c|}{ HH: before direct contact with the patient } \\
\hline Physicians & 82 & 16 & $19.51(10.93-28.09)$ & 96 & 6 & $6.25(1.41-11.09)$ \\
\hline Nurses & 105 & 34 & $32.38(23.43-41.33)$ & 157 & 27 & $17.20(11.29-23.10)$ \\
\hline Nurse aide & 12 & 6 & $50.00(21.71-78.29)$ & 47 & 13 & $27.66(14.87-40.45)$ \\
\hline \multicolumn{7}{|c|}{ HH: after direct contact with the patient } \\
\hline Physicians & 72 & 37 & $51.93(39.84-62.93)$ & 80 & 34 & $42.50(31.67-53.33)$ \\
\hline Nurses & 133 & 101 & $75.94(68.68-83.20)$ & 146 & 61 & $41.78(33.78-49.78)$ \\
\hline Nurse aide & 25 & 24 & $96(88.32-100.00)$ & 31 & 12 & $38.71(21.56-55.86)$ \\
\hline \multicolumn{7}{|c|}{ HH: after removing the gloves } \\
\hline Physicians & 70 & 41 & $58.57(47.03-70.11)$ & 24 & 12 & $50.00(30.00-70.00)$ \\
\hline Nurses & 212 & 125 & $58.96(52.34-65.58)$ & 116 & 43 & $37.07(28.28-45.86)$ \\
\hline Nurse aide & 28 & 21 & $75(58.96$ - 91.04) & 46 & 17 & $36.96(23.01-50.91)$ \\
\hline \multicolumn{7}{|c|}{$\mathrm{HH}$ : before using an invasive device for patient care } \\
\hline Physicians & 27 & 12 & $44.44(25.70-63.19)$ & 8 & 0 & $00.00(/)$ \\
\hline Nurses & 20 & 9 & $45.00(23.20-66.80)$ & 14 & 1 & $7.14(0.00-20.63)$ \\
\hline Nurse aide & 1 & 1 & $100.00(100.00-100.00)$ & not recorded & not recorded & - \\
\hline \multicolumn{7}{|c|}{ HH: after contact with inanimate objects including medical equipment } \\
\hline Physicians & 39 & 5 & $12.82(2.33-23.31)$ & 33 & 10 & $30.30(14.62-45.98)$ \\
\hline Nurses & 110 & 29 & $26.36(18.13-34.60)$ & 92 & 22 & $23.91(15.20-32.63)$ \\
\hline Nurse aide & 7 & 4 & $57.14(20.48-93.80)$ & 17 & 5 & $29.41(7.75-51.07)$ \\
\hline \multicolumn{7}{|c|}{ Use gloves: for assistance patients } \\
\hline Physicians & 122 & 62 & $50.82(41.95-59.69)$ & 63 & 24 & $38.10(26.10-50.09)$ \\
\hline Nurses & 259 & 187 & $72.20(66.74-77.66)$ & 159 & 116 & $10.06(5.39-14.74)$ \\
\hline Nurse aide & 25 & 23 & $92.00(81.37-100.00)$ & 50 & 50 & $100.00(100.00-100.00)$ \\
\hline \multicolumn{7}{|c|}{ Discharge gloves: after contact with the patient } \\
\hline Physicians & 53 & 42 & $79.25(68.33-90.16)$ & 35 & 20 & $57.14(40.75-73.54)$ \\
\hline Nurses & 171 & 137 & $80.12(74.13-86.10)$ & 68 & 66 & $97.6(93.04-100.00)$ \\
\hline Nurse aide & 17 & 12 & $70.59(48.93-92.25)$ & 71 & 56 & $78.87(69.38-88.37)$ \\
\hline \multicolumn{7}{|c|}{ Use of the same gloves to assist more than one patient } \\
\hline Physicians & 36 & 0 & $00.00(/)$ & 6 & 4 & $66.67(28.95-100.00)$ \\
\hline Nurses & 113 & 2 & $1.77(0.00-4.20)$ & 24 & 17 & $70.83(52.65-89.02)$ \\
\hline Nurse aide & 9 & 0 & $00.00(/)$ & not recorded & not recorded & 1 \\
\hline \multicolumn{7}{|c|}{$\begin{array}{l}\text { Discharge gloves: after touching environmental surfaces in proximity of the patient, } \\
\text { including medical equipment }\end{array}$} \\
\hline Physicians & 14 & 6 & $42.86(16.93-68.78)$ & 27 & 11 & $40.74(22.21-59.27)$ \\
\hline Nurses & 107 & 46 & $42.99(33.61-52.37)$ & 29 & 15 & $51.72(33.54-69.91)$ \\
\hline Nurse aide & 12 & 8 & $66.67(39.99-93.34)$ & 7 & 5 & $71.43(37.96-100.00)$ \\
\hline
\end{tabular}

${ }^{\mathrm{a}}$ Values are expressed as numerical frequencies unless otherwise indicated.

problems with this method can include the Hawthorne effect (16) (e.g., process in which individuals change or improve their behavior in response to their awareness of being observed), as well as the fact that only a small fraction of hand hygiene opportunities can be observed. To try to prevent or reduce the Hawthorne effect, in our study the operators were observed as many times as possible. In this way, the observed worker had the opportunity to feel comfortable with the presence of the observers, thus reducing the likelihood for bias due to their presence.
The widespread knowledge and compliance to preventive measures can represent one of the main tools for the prevention of nosocomial HAV infections and other nosocomial infections that pose a risk for both patients and workers. Due to the reduction in the circulation of HAV, vaccination against HAV should be considered only for those students who are anti-HAV IgG negative and work in wards in which patients with hepatic and infectious diseases are hospitalized, and patients from high endemicity areas (i.e. health services for immigrants) are admitted. Be- 
cause of hepatitis A vaccine's high immunogenic properties $(17,18)$ a single-dose vaccine may be sufficient to protect susceptible individuals exposed to hepatitis A (19).

Surveillance of HAV infection based solely on referring symptomatic cases, who seek medical care, could underestimate the risk (11). Besides, when large outbreaks of hepatitis A occur, disease control costs are higher than direct medical costs (20).

This paper highlights the need for standardized and continuous training for healthcare workers to be delivered by universities and healthcare services in order to ensure that both nursing students and healthcare workers have comprehensive knowledge of the infectious disease to reduce the infection risk.

\section{Footnote}

Authors' Contribution: Marcello Campagna, Noemi Maria Mereu, Igor Portoghese, Maura Galletta, Alberto Lai, Giuseppina Masia, Angelo Restivo, Paolo Mura, Gabriele Finco and Rosa Cristina Coppola: study concept and design, critical revision of the manuscript for important intellectual content and drafting of the manuscript; Marcello Campagna and Rosa Cristina Coppola: study supervision; Noemi Maria Mereu, Lucia Mulas, Roberta Pilia, Maria Francesca Piazza, Laura Spada and Alberto Lai: acquisition of data, statistical analysis, analysis and interpretation of data.

\section{References}

1. Franco E. Hepatitis B: Epidemiology and prevention in developing countries. World J Gastroenterol. 2012;4(3):74. doi:10.4254/wjh.v4.i3.74.

2. Hadler SC. Global impact of hepatitis A virus infection: changing patterns. hepatitis. 1991;1420.

3. Pontrelli G, Boccia D, D. I. Renzi M, Massari M, Giugliano F, Celentano LP, et al. Epidemiological and virological characterization of a large community-wide outbreak of hepatitis A in southern Italy. Epidemiol Infect. 2008;136(8):1027-34. doi: 10.1017/S095026880700951X. [PubMed: 17892633].

4. Campagna M, Siddu A, Meloni A, Basciu C, Ferrai L, Pettinau A, et al. Changing pattern of hepatitis a virus epidemiology in an area of high endemicity. Hepat Mon. 2012;12(6):382-5. doi:10.5812/hepatmon.5940. [PubMed: 22879827].

5. Gibas A, Blewett DR, Schoenfeld DA, Dienstag JL. Prevalence and incidence of viral hepatitis in health workers in the prehepatitis B vaccination era. Am J Epidemiol. 1992;136(5):603-10. [PubMed: 1442723].

6. Kashiwagi S, Hayashi J, Ikematsu H, Nomura H, Kajiyama W, Ikematsu $\mathrm{W}$, et al. Prevalence of immunologic markers of hepatitis A and B infection in hospital personnel in Miyazaki Prefecture, Japan. Am J Epidemiol. 1985;122(6):960-9. [PubMed: 4061446].

7. Pittet D. Hand hygiene: improved standards and practice for hospital care. Curr Opin Infect Dis. 2003;16(4):327-35. doi: 10.1097/01.qco.0000083564.72029.27. [PubMed: 12861085].

8. Grayson ML, Jarvie LJ, Martin R, Johnson PD, Jodoin ME, McMullan C, et al. Significant reductions in methicillin-resistant Staphylococcus aureus bacteraemia and clinical isolates associated with a multisite, hand hygiene culture-change program and subsequent successful statewide roll-out. Med JAust. 2008;188(11):633-40. [PubMed:18513171].

9. Rosenthal VD, Guzman S, Safdar N. Reduction in nosocomial infection with improved hand hygiene in intensive care units of a tertiary care hospital in Argentina. Am J Infect Control. 2005;33(7):392-7. doi: 10.1016/j.ajic.2004.08.009. [PubMed: 16153485].

10. Allegranzi B, Pittet D. Role of hand hygiene in healthcareassociated infection prevention. J Hosp Infect. 2009;73(4):305-15. doi: 10.1016/j.jhin.2009.04.019. [PubMed:19720430].

11. Garus-Pakowska A. [Workload impact on compliance with hygiene procedures in medical personnel]. Med Pr. 2011;62(4):369-76. [PubMed: 21995106].

12. Harbarth S, Sudre P, Dharan S, Cadenas M, Pittet D. Outbreak of Enterobacter cloacae related to understaffing, overcrowding, and poor hygiene practices. Infect Control Hosp Epidemiol. 1999;20(9):598-603. doi: 10.1086/501677. [PubMed: 10501256].

13. Loulergue $P$, Launay $O$. Vaccinations among medical and nursing students: coverage and opportunities. Vaccine. 2014;32(38):4855-9. doi: 10.1016/j.vaccine.2014.01.014. [PubMed: 24503273].

14. Boyce JM, Pittet D. Guideline for hand hygiene in health-care settings: recommendations of the Healthcare Infection Control Practices Advisory Committee and the HICPAC/SHEA/APIC/IDSA Hand Hygiene Task Force. Infect Control Hosp Epidemiol. 2002;30(8):S1-S46.

15. Field DE. Moving from novice to expert - the value of learning in clinical practice: a literature review. Nurse Educ Today. 2004;24(7):560-5. doi: 10.1016/j.nedt.2004.07.009. [PubMed:15465172].

16. McCambridge J, Witton J, Elbourne DR. Systematic review of the Hawthorne effect: new concepts are needed to study research participation effects. J Clin Epidemiol. 2014;67(3):267-77. doi: 10.1016/j.jclinepi.2013.08.015. [PubMed: 24275499].

17. Andre F, Van Damme P, Safary A, Banatvala J. Inactivated hepatitis A vaccine: immunogenicity, efficacy, safety and review of official recommendations for use. Expert Rev Vaccines. 2002;1(1):9-23. doi: 10.1586/14760584.1.1.9. [PubMed: 12908508].

18. Ansaldi F, Bruzzone B, Rota MC, Bella A, Ciofi degli Atti M, Durando $P$, et al. Hepatitis A incidence and hospital-based seroprevalence in Italy: a nation-wide study. Eur J Epidemiol. 2008;23(1):45-53. doi: 10.1007/s10654-007-9198-y. [PubMed: 17978852].

19. Ott JJ, Wiersma ST. Single-dose administration of inactivated hepatitis A vaccination in the context of hepatitis A vaccine recommendations. Int J Infect Dis. 2013;17(11):e939-44. doi: 10.1016/j.ijid.2013.04.012. [PubMed: 23791857].

20. Rosenthal P. Cost-effectiveness of hepatitis A vaccination in children, adolescents, and adults. Hepatology. 2003;37(1):44-51. doi: 10.1053/jhep.2003.50016. [PubMed: 12500187]. 